\title{
Inter-relationships of Alleles at the am Locus in Neurospora crassa
}

\author{
By J. A. PATEMAN* \\ Botany School, University of Melbourne, Australia
}

(Received 16 May 1960)

\begin{abstract}
SUMMARY
It has been shown that the alleles 5, 6 and 7 at the am locus in Neurospora crassa backmutate; the alleles $4,8,9$ and 11 do not backmutate at detectable frequencies. Wild-types were recovered in low frequencies from crosses between different am alleles. It has been shown that each of the twelve am alleles, so far investigated, is unique with respect to at least one of the following criteria: mutation, recombination, complementation, type of glutamic dehydrogenase produced. It is argued that the minimum number of possible mutations at the am locus, which can affect glutamic dehydrogenase production, is eighteen. The probability that this minimum estimate is too large is less than $1 \%$.
\end{abstract}

\section{INTRODUCTION}

It is known that mutations at the 'amination deficient', am, locus in Neurospora crassa specifically affect the formation of the enzyme glutamic dehydrogenase by the organism (Fincham, 1954; Pateman \& Fincham, 1958). A number of independently induced am mutants have recently become available. This paper is chiefly concerned with the inter-relationships between ten independently induced am mutants as determined by the criteria of complementation, mutation and recombination.

\section{METHODS}

Experimental strains. The origin of the am strains and of the stock wild types was described previously (Pateman \& Fincham, 1958; Fincham, 1959). The symbols used for the am mutants in the present paper differ from those used by Pateman \& Fincham (1958). The superscripts, 32, 47, 29 and $R$, are replaced and all the am strains are now specified by a simple numerical superscript forming the series $a m^{1}$ to $a m^{11}$. For the sake of clarity, $a m$ strains will usually be referred to by number only. For example, $a m^{2}$ would be denoted by 2 , the locus symbol am being understood.

Culture media. For growth tests and stock cultures Fries No. 3 minimal medium was used, with suitable supplements where necessary. In the backmutation experiments the usual energy source, $1.5 \%(\mathrm{w} / \mathrm{v})$ sucrose, was replaced by $1.5 \%$ sorbose $+\mathbf{0 . 7 5} \%$ sucrose. On this media Neurospora crassa has a colonial growth habit. All crosses between strains were made at $25^{\circ}$ on a medium favouring sexual reproduction (Westergaard \& Mitchell, 1947).

* Present address : Department of Genetics, University of Cambridge. 
Experimental procedure in irradiation experiments. The details of the backmutation experiments were similar to those previously described by Pateman (1957), with the following exceptions. The source of ultraviolet radiation was a General Electric Co. (G-15 T-8), $15 \mathrm{w}$ low-pressure mercury-vapour germicidal lamp. During irradiation the conidial suspension formed a layer approximately $3.0 \mathrm{~mm}$. thick in an open Petri dish. The surface of the suspension was approximately $8.0 \mathrm{~cm}$. from the lamp; under these conditions an exposure time of $90 \mathrm{sec}$. killed $99.0 \%$ of the conidia.

\section{RESULTS \\ Backmutation of am alleles}

It was shown previously (Pateman, 1957) that the am strains 1, 2 and 3 will backmutate as a result of ultraviolet irradiation. Similar series of backmutation experiments were carried out with strains 4, 5, 6, 7, 8,9 and 11. Macroconidia were collected from 14-day cultures grown at $25^{\circ}$, then irradiated for $90 \mathrm{sec}$. to give a c. $99 \%$ kill, and spread on Fries medium + sorbose + glycine agar contained in Petri dishes. The survival rate of conidia after irradiation varied from 0.5 to $4 \%$ in different experiments, but in the majority was close to $1 \%$. The number of live am conidia after irradiation in each experiment was usually between $0.5 \times 10^{6}$ and $1.5 \times 10^{6}$. The total number of backmutation experiments with the seven am alleles was thirty-eight. The results of these experiments are given in Table 1. After incubation at $25^{\circ}$ for $72-90 \mathrm{hr}$. any wild-type colonies which appeared were transferred to minimal medium slopes. The resultant cultures were subsequently tested for growth on minimal medium to check their apparent wild phenotype.

Table 1. Frequency of backmutation in certain am alleles in

Neurospora crassa

$\begin{array}{cccc}\text { am } \text { allele } & \begin{array}{c}\text { Total no. of } \\ \text { live conidia } \\ \left(\times 10^{-6}\right)\end{array} & \begin{array}{c}\text { Total no. of } \\ \text { wild types }\end{array} & \begin{array}{c}\text { Mean backmutation } \\ \text { rate per } 10^{6} \\ \text { live conidia }\end{array} \\ 4 & 11 \cdot 71 & 1 & - \\ 5 & 1 \cdot 29 & 26 & c .20 \cdot 0 \\ 6 & 1 \cdot 40 & 37 & c .26 \cdot 4 \\ 7 & 10 \cdot 80 & 21 & c .1 \cdot 9 \\ 8 & 4 \cdot 86 & 0 & - \\ 9 & 8 \cdot 97 & 0 & - \\ 11 & 7 \cdot 71 & 0 & -\end{array}$

It is clear from Table 1 that the am alleles fell into two distinct groups. One group contained 4, 8, 9 and 11, all of which did not backmutate at a detectable frequency. One wild-type colony was in fact recovered from experiments with 4 . This might have been a contaminant, but if genuine, then the backmutation frequency of 4 was certainly much lower than that of the 5, 6 and 7 alleles. The other group comprised 5,6 and 7 which did backmutate at frequencies sufficiently high to be easily detected. The approximate backmutation frequencies obtained for 5 and 6 are not significantly different. The frequency found for 7 is significantly different from both 5 and 6 ; comparison of 7 with 5 and 6 combined gives a value of $t_{10}=7 \cdot 621, P<0.001$.

The characteristic backmutation frequency of each am allele might be a specific property of the allele itself or, at least in part, a reflexion of the background 
genotype. Each of the am strains used in these experiments had been backcrossed at least three and usually four or more times to the same stock strain, St Lawrence wild type (Pateman, 1957). Therefore the stocks were largely isogenic and it is probable that the large difference in backmutation frequencies between the two groups of alleles was due to specific characteristics of the alleles themselves. The significant difference in backmutation frequency between 5 and 6 and the other allele in that group (7) also probably reflects different specific properties of the actual alleles. There are two main points established by these experiments. The am alleles 5, 6 and 7 are sharply distinguished from 4, 8, 9 and 11 by the criterion of backmutation. The genetic change which results in the manifestation of the am phenotype in 5, 6 and 7 cannot be a deletion, since these strains readily backmutate.

\section{Recombination of am alleles}

It is known (Pateman, 1958, 1960) that true wild types can be recovered from crosses between $a m^{2}$ and $a m^{3}$ with a frequency of about 1/50,000 live ascospores. A further series of experiments in which crosses between different am alleles were analysed with respect to the production of wild types is described here. The experimental details are similar to those previously described by Pateman (1958) and consisted essentially of plating ascospores from $a m \times a m$ crosses on a glycine medium on which the $\mathrm{am}^{+}$ascospores had a strong selective advantage. Apparent $\mathrm{am}^{+}$ascospores were isolated, cultured and subsequently tested in growth flasks to confirm that they were phenotypically wild-type. 'The results of these experiments are given in Table 2; the results given in line 1 are from Pateman $\&$ Fincham (1958)

\begin{tabular}{|c|c|c|c|}
\hline Cross & $\begin{array}{c}\text { No. of } \\
\text { wild types }\end{array}$ & $\begin{array}{l}\text { No. of live } \\
\text { ascospores } \\
\text { (thousands) }\end{array}$ & $\begin{array}{l}\text { Frequency of } \\
\text { wild types } \\
\text { per } 10^{6} \text { live } \\
\text { ascospores }\end{array}$ \\
\hline $2 \times 3$ & 104 & $544: 3$ & $19 \cdot 1$ \\
\hline $4 \times 8$ & 8 & 513 & $16 \cdot 0$ \\
\hline $4 \times 9$ & 22 & 305 & $72 \cdot 0$ \\
\hline $4 \times 11$ & 14 & 164 & $85 \cdot 0$ \\
\hline $5 \times 6$ & 9 & 715 & $13 \cdot 0$ \\
\hline $5 \times 7$ & 1 & 1355 & 0.75 \\
\hline $6 \times 7$ & 9 & 586 & $15 \cdot 0$ \\
\hline $8 \times 9$ & 4 & 566 & $7 \cdot 1$ \\
\hline $8 \times 11$ & 25 & 504 & $50 \cdot 0$ \\
\hline $9 \times 11$ & 3 & 712 & 4.2 \\
\hline
\end{tabular}

and Pateman (1960). It has been shown (Pateman \& Fincham, 1958) that wild-types are not recovered from crosses between identical am parents. Therefore the data in Table 2 show that recombination occurred between the pairs of am strains listed, with the exception of 5 and 7 . In the latter case recombination was doubtful since only one wild type was recovered.

\section{Differentiation of am alleles}

There are four criteria available for differentiating between the known am alleles, namely, mutation, recombination, complementation, and type of enzyme produced. 
Mutation and recombination have been used in previous sections. It is necessary at this point to outline the situation with regard to complementation. It has been shown (Fincham \& Pateman, 1957 a; Pateman \& Fincham, 1958; Fincham, 1959) that certain combinations of am alleles in heterocaryons were complementary with respect to the production of glutamic dehydrogenase. The only combinations of alleles from the present series which were complementary were $1+2$ and $1+3$. Either of these pairs of alleles gave heterocaryons which grew on minimal medium without lag and yielded detectable glutamic dehydrogenase activities up to about $25 \%$ of that normally found in wild-type.

In addition to the $a m$ alleles which result in a complete lack of detectable glutamic dehydrogenase activity, a number of secondary mutant alleles are known (Pateman, 1957). These are backmutations at the am locus which restore some glutamic dehydrogenase activity, but not the normal amount found in wild-type strains. Two of these unusual backmutants have been further investigated (Fincham, 1957; Fincham \& Pateman 1957 $b$; P. Bond, unpublished; J.R.S. Fincham, unpublished) and shown to possess a qualitatively different glutamic dehydrogenase both from wild-type and from each other. So far, only one of these strains, designated $a m^{2 l}$, has been tested for complementation with the other alleles (Fincham, 1959).

\section{Table 3. Relationships between am alleles in Neurospora crassa}

\begin{tabular}{|c|c|c|c|c|}
\hline & $\begin{array}{l}\text { Alleles which result } \\
\text { in the production } \\
\text { of a qualitatively } \\
\text { different glutamic } \\
\text { dehydrogenase }\end{array}$ & $\begin{array}{l}\text { Combinations of } \\
\text { alleles which show } \\
\text { complementation }\end{array}$ & $\begin{array}{l}\text { Alleles which } \\
\text { backmutate }\end{array}$ & $\begin{array}{c}\text { Alleles which do } \\
\text { not backmutate } \\
\text { or show } \\
\text { complementation }\end{array}$ \\
\hline & $\begin{array}{c}21 \\
1038^{*}\end{array}$ & $\begin{array}{l}1+2,1+3 \\
1+2 l, 3+2 l, \\
4+2 l, 6+2 l \\
7+2 l, 8+2 l \\
9+2 l, 11+2 l\end{array}$ & $\begin{array}{l}1,2,3 \\
5,6,7\end{array}$ & $4,8,9,11$ \\
\hline $\begin{array}{l}\text { Pairs of alleles } \\
\text { which show } \\
\text { recombination }\end{array}$ & -- & $2 \times 3$ & $\begin{array}{l}2 \times 3,5 \times 6, \\
5 \times 7 ?, 6 \times 7\end{array}$ & $\begin{array}{l}4 \times 8,4 \times 9 \\
4 \times 11,8 \times 9 \\
8 \times 11,9 \times 11\end{array}$ \\
\hline
\end{tabular}

Table 3 is a summary of all the relevant information concerning the am alleles arranged to illustrate the point that each of the twelve so far investigated is unique. The alleles $2 l$ and 1038 are clearly distinct from each other and all the rest since they result in the production of qualitatively different enzymes. Within the series 1-11 each allele can be shown to be unique as follows. Only 1 is complementary with 2 and 3 and is therefore distinct from all the rest. Since only 2 and 3 are complementary with 1 they form a separate group, but 2 and $\mathbf{3}$ are distinct since they show recombination. The alleles $1,2,3,5,6$ and 7 form a separate group since they backmutate; within this group recombination indicates that each allele is unique with the possible exception of 5 and 7 . Since only one wild-type was recovered from the cross $5 \times 7$, recombination is doubtful. But 7 is complementary with 21 and 5 is not, also 7 gave a significantly different backmutation frequency from 5 , therefore 5 and 7 are different. The alleles 4, 8, 9 and 11 form a separate 
group since they do not backmutate at a detectable frequency. Recombination shows that each member of this group is distinct from the rest. Thus it is clear that each of the twelve alleles is different from the rest with respect to at least one of the four criteria which can be used to differentiate between them.

\section{Number of possible kinds of mutation at the am locus}

It is of interest to attempt to determine an estimate of the minimum number of sites which can be altered by mutation in a complex locus. Such estimates have been derived for adenine loci in Saccharomyces (Roman, 1956), for histidine loci in Salmonella (Hartman, Hartman \& Šerman, 1960) and for complex loci in Drosophila, Aspergillus, bacteriophage and Schizosaccharomyces (Pontecorvo, 1958). In all these cases the method of estimation depends essentially on recombination data. In the case of the am locus in Neurospora crassa the situation is somewhat different. The first twelve alleles investigated were derived from five independent sources, ten were induced by ultraviolet radiation, one by 20 -methylcholanthrene and one by $\beta$-propiolactone. Each of the twelve is unique as judged by four independent criteria, of which recombination is one. If it be assumed that they approach a random sample, then an estimate of the minimum number of possible mutations at the $a m$ locus which can affect glutamic dehydrogenase production may be made. It should be noted that the estimate concerns the minimum number of different kinds of mutation at the am locus which can affect glutamic dehydrogenase production, not the minimum number of mutational sites. It is possible that more than one kind of mutation can occur at a mutational site within the locus. The minimum estimates made by other workers, referred to above, concern mutational sites separable by recombination, since the estimates were essentially based on recombination data only.

The argument on which the estimate of the minimum possible number of am alleles rests is as follows. Assume that the possible kinds of mutation at the am locus are equally likely. The question of estimation then concerns the probable size of the population of different, equally likely, kinds of mutation from which a random sample of twelve prove to be all different. It can readily be shown, if a random sample of size $r$ is taken from a population made up of $n$ kinds of event, that $\boldsymbol{P}$, the probability all $r$ will be different is given by

$$
P=\frac{n !}{n^{r}(n-r) !} .
$$

In the present case $r$ is the number of different alleles, $n$ the number of different kinds of mutation. For $r=12, n=26, P=0.04843$. For $r=12, n=18$, $P=0.007679$. The latter set of conditions is equivalent to the following statement. Since each of the first twelve alleles investigated is unique, there are at least eighteen different possible kinds of mutation at the am locus, and the probability that this estimate is too large is less than $1 \%$. Similarly it can be stated that the minimum number of possible kinds of mutation is twenty-six, with a probability that this estimate is too large of less than $5 \%$. The estimates are minimum ones for the appropriate probability levels. The actual number of possible kinds of mutation may be higher. 
It is possible, indeed probable, that the assumption that all the possible kinds of mutation are equally likely, is incorrect. This would mean that one (or more) of the possible kinds of mutation was more frequent than the rest. Such a relatively frequent mutation would stand a proportionally greater chance of being included twice in the random sample, whatever the size of the latter. Consequently, the existence of a relatively frequent mutation can only decrease the average size of the sample which contains only non-identical mutations. That is, the existence of one or more relatively frequent mutations could only decrease the estimate of the minimum number of possible kinds of mutation. Thus the possibility of different relative mutation frequencies does not invalidate the estimates of the minimum number of possible kinds of mutation at the am locus.

\section{DISCUSSION}

It is now known that in many respects the am locus in Neurospora crassa is similar to other complex loci which have been investigated in detail. Recombination between $\mathrm{am}$ alleles, at least partly due to a mechanism other than classical crossingover (Pateman, 1958, 1960), complementation, and differences in mutation frequency, have all been demonstrated. These are phenomena commonly associated with complex loci in bacteriophage, bacteria, fungi and higher organisms. The phenomenon of complementation represents a functional aspect of the complex locus. Benzer (1957) proposed the term 'cistron' for the functional unit of the gene. The definition of the cistron is an operational one based on complementation. Several cases are already known which do not fit the cistron concept in its simplest form. The ad-9 locus in Aspergillus (Calef, 1957) and the hisB locus in Salmonella (Demerec \& Hartman, 1959) are examples. In the case of the am locus in Neurospora, neither the whole locus nor any subunit corresponds to a cistron. The fact that certain combinations of alleles show complementation implies the existence of functional subunits within the am locus. But most of the possible combinations of $a m$ alleles are non-complementary. Consequently to fit the cistron concept, the majority of the apparent alleles would need to be regarded as deletions of various sizes (Fincham, 1959). Such a numerical preponderance of deletions over point mutations as a result of ultraviolet irradiation seems unlikely. Also 2, 5, 6 and 7, each of which would cover two or more cistrons, are known to backmutate and thus cannot be deficiencies.

In view of this consideration there appear to be two main possibilities concerning the situation at the am locus in Neurospora. Either the complementary relationships between the alleles represent an aspect of the am locus which is non-linear, or some of the point mutations occur at sites especially critical for the production of an integrated gene product, in this case presumably the enzyme glutamic dehydrogenase. The latter alternative seems, to the author, the most probable. It is similar to a possibility suggested by Demerec \& Hartman (1959) in connexion with a number of alleles, which are at variance with the cistron concept, in the hisB locus in Salmonella typhimurium.

This work was supported in part by a grant from the Anti-Cancer Council of Victoria, Australia. 


\section{REFERENCES}

Benzer, S. (1957). The elementary units of heredity. In $A$ Symposium on the Chemical Basis of Heredity, ed. W. D. McElroy and B. Glass, pp. 70. Baltimore: The Johns Hopkins Press.

CALEF, E. (1957). Effect on linkage maps of selection of crossovers between closely linked markers. Heredity, 12, 265.

Demerec, M. \& Hartman, P. E. (1959). Complex loci in micro-organisms. Annu. Rev. Microbiol. 13, 377.

Fincham, J. R. S. (1954). Effects of a gene mutation in Neurospora crassa relating to glutamic dehydrogenase formation. J. gen. Microbiol. 11, 236.

Fincham, J. R. S. (1957). A modified glutamic acid dehydrogenase as a result of gene mutation in Neurospora crassa. Biochem. J. 65, 721.

Fincham, J. R. S. (1959). The role of chromosomal loci in enzyme formation. Proc. 10th Congr. Genet. 1, 355.

Fincham, J. R. S., \& Pateman, J. A. (1957 a). Formation of an enzyme through complementary action of mutant alleles in separate nuclei in a heterocaryon. Nature, Lond. 179, 741.

Fincham, J. R. S. \& Pateman, J. A. (1957 b). A new allele at the am locus of Neurospora crassa. J. Genet. 55, 456.

Hartman, P. E., Hartman, Z., \& Šerman, D. (1960). Complementation mapping by abortive transduction of histidine-requiring Salmonella mutants. J. gen. Microbiol. 22, 354.

Pateman, J. A. (1957). Backmutation studies at the am locus in Neurospora crassa. J. Genet. 55, 444.

Pateman, J. A. (1958). Aberrant recombination at the am locus in Neurospora crassa. Nature, Lond. 181, 1605.

Pateman, J. A. (1960). High negative interference at the am locus in Neurospora crassa. Genetics (in the Press).

Pateman, J. A. \& Fincham, J. R. S. (1958). Gene-enzyme relationships at the am locus in Neurospora crassa. Heredity, 12, 317.

Ponteconvo, G. (1958). Trends in Genetic Analysis, 1st ed. New York: Columbia University Press.

Roman, H. (1956). Studies of gene mutation in Saccharomyces. Cold Spr. Harb. Symp. quant. Biol. 21, 175.

WestergaArd, M. \& Mitchell, H. K. (1947). Neurospora V. A synthetic medium favouring sexual reproduction. Amer. J. Bot. 34, 573. 